\title{
Identification of specifically activated angiogenic molecules in HMGB-1-induced angiogenesis
}

\author{
Won Kyu Kim,"\#, Yujin Kwon ${ }^{1, \#}$, Minhee Park ${ }^{1}$, Seongju Yun ${ }^{1}$, Ja-Young Kwon ${ }^{2}$ E Hoguen Kim ${ }^{1, *}$ \\ ${ }^{1}$ Department of Pathology and Brain Korea 21 PLUS Projects for Medical Science, Yonsei University College of Medicine, Seoul 03722, \\ ${ }^{2}$ Departments of Obstetrics and Gynecology, Institute of Women's Life Medical Science, Yonsei University College of Medicine, Seoul \\ 03722, Korea
}

\begin{abstract}
High-mobility group box-1 (HMGB-1) is expressed in almost all cells, and its dysregulated expression correlates with inflammatory diseases, ischemia, and cancer. Some of these conditions accompany HMGB-1-mediated abnormal angiogenesis. Thus far, the mechanism of HMGB-1-induced angiogenesis remains largely unknown. In this study, we performed time-dependent DNA microarray analysis of endothelial cells (ECs) after HMGB-1 or VEGF treatment. The pathway analysis of each gene set upregulated by HMGB-1 or VEGF showed that most HMGB-1-induced angiogenic pathways were also activated by VEGF, although the activation time and gene sets belonging to the pathways differed. In addition, HMGB-1 upregulated some VEGFR signaling-related angiogenic factors including EGR1 and, importantly, novel angiogenic factors, such as ABL2, CEACAM1, KIT, and VIPR1, which are reported to independently promote angiogenesis under physiological and pathological conditions. Our findings suggest that HMGB-1 independently induces angiogenesis by activating HMGB-1-specific angiogenic factors and also functions as an accelerator for VEGF-mediated conventional angiogenesis. [BMB Reports 2017; 50(11): 590-595]
\end{abstract}

\section{INTRODUCTION}

The highly conserved protein high-mobility group box-1 (HMGB-1) plays various roles according to cellular localization and is expressed in almost all mammalian cells $(1,2)$. Secreted HMGB-1 functions as an immune cell-associated cytokine that induces systemic inflammation, cell proliferation,

${ }^{*}$ Corresponding author. Tel: +82-2-2228-1761; Fax: +82-2-3635263; E-mail: hkyonsei@yuhs.ac

${ }^{\#}$ These authors contributed equally to this work.

https://doi.org/10.5483/BMBRep.2017.50.11.129

Received 12 July 2017, Revised 2 August 2017, Accepted 29 September 2017

Keywords: Angiogenesis, Endothelial cell, Gene expression profile, HMGB-1, VEGF signal transduction, and activation of endothelial cells (ECs) (3). Secreted HMGB-1 activates downstream signaling pathways (CDC42/Rac, MAPK, NF- $\mathrm{KB}$, and PI3K-Akt) by binding to several known receptors, such as the receptor for advanced glycation end products (RAGE) and the Toll-like receptors TLR-2, TLR-4, and TLR-9 (4-7).

The pathogenic functions of HMGB-1 have been documented in a wide range of diseases such as neurodegeneration, aging, ischemia, and cancers (8-10). In particular, HMGB-1 overexpression is now considered a hallmark of various types of cancers including colon, breast, and lung cancer and hepatocellular carcinoma, and its expression correlates with invasion, metastasis, and poor prognosis (11). Therefore, HMGB-1-targeted cancer therapeutic strategies using siRNA and antibodies have been developed and applied to in vitro and in vivo disease models $(12,13)$. HMGB-1 inhibition by these agents potently suppresses the progression of cancers through the perturbations of migration, proliferation, and angiogenesis $(7,14)$. The inhibition of angiogenesis is particularly important in advanced cancers because angiogenesis is a crucial step for the acquisition of malignant properties such as metastasis and invasion (15). While HMGB-1-mediated angiogenesis is disturbed by siRNA-mediated downregulation of RAGE or by HMGB-1 neutralizing antibody $(16,17)$, the mechanisms of HMGB-1-mediated angiogenesis are not fully understood in terms of the transcriptomic changes caused by exogenous HMGB-1 stimulation. Moreover, the differences between HMGB-1-mediated angiogenesis and conventional VEGF-mediated angiogenesis are essentially unknown.

In this study, we aimed to characterize the angiogenic pathways and genes activated in ECs treated with HMGB-1 or VEGF at various time points and how they contribute to angiogenesis. We found that HMGB-1 as well as VEGF significantly induced proangiogenic features in ECs, accompanying time-dependent activation of angiogenic pathways. HMGB-1 treatment also resulted in HMGB-1-specific upregulation and VEGF-like upregulation of oncogenic and angiogenic genes. Our findings indicate that HMGB-1 can function as a potent angiogenic signal both independently and synergistically in combination with other angiogenic signals. 


\section{RESULTS}

\section{HMGB-1 treatment confers proangiogenic features on ECs}

Given the expected roles of HMGB-1 in angiogenesis, we first tested whether exogenous HMGB-1 treatment stimulated proangiogenic features in ECs by evaluating proliferation, migration, wound healing, and tube formation after HMGB-1 treatment in a time-dependent manner. ECs were isolated from human placental microvessels and validated by analyzing CD31 expression using fluorescence-activated cell sorting (FACS; data not shown). ECs were counted after 48 hours of HMGB-1 or VEGF treatment, and relative proliferation was measured. HMGB-1 treatment induced an approximately twofold increase in EC proliferation compared to that of VEGF treatment (Fig. 1A). The migration assay also showed that HMGB-1 treatment increased migrated EC numbers 2.5-fold (Fig. 1B). The wound healing assay to further evaluate both migration and cell-cell interaction showed that wound healing was significantly promoted by HMGB-1 treatment (Fig. 1C and D) and that HMGB-1-treated ECs were more likely to form capillary-like structures (Fig. 1E and F). Although VEGFmediated proangiogenic activation of ECs is well known, we further confirmed that exogenous VEGF treatment strongly induced the proangiogenic features of ECs in our system (Fig. 1A-F).

\section{Microarray profiling of gene expression in ECs activated by HMGB- 1 or VEGF}

We performed gene expression microarray analysis to clarify
A

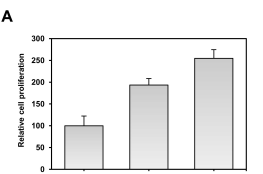

c

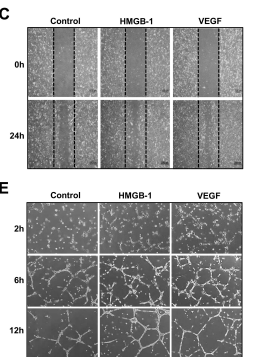

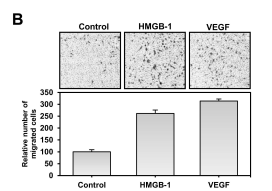

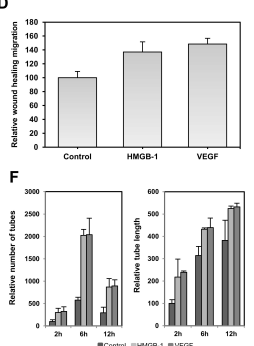

Fig. 1. HMGB-1 and VEGF stimulate proangiogenic features in ECs. (A) Proliferation assay. ECs were counted after 48 hours of HMGB-1 or VEGF treatment. (B) Invasion assay. Migrated ECs were stained and counted after 18 hours of treatment with HMGB-1 or VEGF. (C and D) Wound healing assay. Relative wound healing rates were measured in ECs after wound creation and 24 hours of treatment with HMGB-1 or VEGF. (E and F) Tube formation assay. The relative number of tubes and tube length were measured manually after ECs were incubated on Martrigel with HMGB-1 or VEGF for 2, 6, and 12 hours. the molecular mechanism of HMGB-1-induced EC activation at the gene expression level. ECs were treated with HMGB-1 or VEGF at various time points to investigate time-dependent gene expression changes. RNAs were extracted from ECs harvested at early time points (20 and 45 minutes after HMGB-1 or VEGF treatment) and late time points $(2,6$, and 12 hours after HMGB-1 or VEGF treatment), and gene expression microarray profiling was performed. By pre-processing the raw data, we obtained 19,389 and 19,335 probes in HMGB-1treated and VEGF-treated samples, respectively. Further selection of probes that showed more than 1.5-fold up- or downregulation resulted in 507 probes in HMGB-1-treated samples and 1,212 probes in VEGF-treated samples (Fig. 2A). Selected probes were mapped to official genes and K-means, and hierarchical clustering was performed using the list of obtained genes. K-means cluster analysis showed that gene expression was distinctively altered in a time-dependent manner in ECs treated with HMGB-1 compared to that in ECs treated with VEGF. Gene expression patterns changed dramatically between 45 minutes and 2 hours after HMGB-1 treatment, while VEGF treatment induced more gradual gene expression changes (Fig. 2B). To validate our microarray data, eight genes that showed distinct expression levels at each time point after HMGB-1 or VEGF treatment were selected (CCL2, CCND2, EPHB1, PCDH12, VASH1, TMEM140, CD34, and $E G R 1)$, and qRT-PCR was performed in another set of EC samples treated with HMGB-1 or VEGF in the same manner as in the microarray analysis. To accurately compare our microarray data with qRT-PCR data, expression intensities of

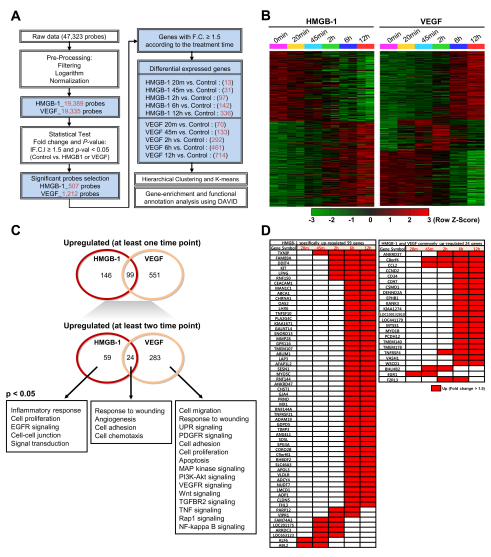

Fig. 2. HMGB-1 induces angiogenic pathways via genes specifically activated by HMGB-1 and those activated by both HMGB-1 and VEGF. (A) Analysis pipeline of time-dependent gene expression microarray performed in ECs treated with HMGB-1 or VEGF. (B) K-means cluster analysis in ECs treated with HMGB-1 or VEGF for various periods of time. (C) Ontology analysis of genes upregulated by HGMB-1 or VEGF during at least two time points. (D) Time-dependent expression patterns of genes specifically upregulated by HMGB-1 $(\mathrm{n}=59)$ and those upregulated by both HMGB-1 and VEGF $(\mathrm{n}=24)$. 
the eight genes obtained from our microarray data were normalized to $\beta$-actin intensities. Expression patterns as determined by qRT-PCR were similar to those observed in the microarray data (Supplementary Fig. S1). However, there were some discrepancies in TMEM140, CD34, and ECR1 between the results from microarray and qRT-PCR in both HMGB-1treated and VEGF-treated samples. We, therefore, further performed qRT-PCR analysis of the three genes using GAPDH, another highly stable housekeeping gene, and compared the results with our microarray data normalized to GAPDH intensities, which allowed us to observe minimal discrepancies between the results from the microarray and qRT-PCR in all three genes (Supplementary Fig. S2A and S2B). Our microarray data were further validated at the protein level. We obtained commercially available antibodies against CCL2, PCDH12, CD34, and EGR1 and performed western blotting analysis with the same EC samples used for the qRT-PCR analysis. Western blotting analysis showed that the mRNA expressions of the four genes were highly proportional to their protein expressions, which validates our microarray data (Supplementary Fig. S2C and S2D).

\section{HMGB-1 and VEGF induce characteristic activation of angiogenic pathways}

The number of genes upregulated by HMGB-1 or VEGF by more than 1.5 -fold gradually increased in a time-dependent manner, with more genes upregulated by VEGF than HMGB-1 at each time point. Using this list of upregulated genes at each time point, we performed gene enrichment and functional annotation analyses with DAVID.

Most upregulated genes after 20 minutes of VEGF treatment belonged to well-documented VEGF-dependent angiogenic pathways, such as the MAP kinase, hypoxia, chemotaxis, cell migration, cell proliferation, apoptosis, angiogenesis, vasculogenesis, tumor necrosis factor (TNF) signaling, and transforming growth factor beta receptor (TGFBR) signaling pathways (3, 18). Most of these pathways remained activated after 45 minutes of VEGF treatment, at which point the unfolded protein response (UPR), cell adhesion, NF- $\mathrm{KB}$ signaling, and cell differentiation pathways were newly activated. No significant changes in the activated pathways were found after 2 hours of VEGF treatment except for the upregulation of some genes related to the canonical Wnt and Notch signaling pathways, which are also known to be involved in vessel branching. Similarly, no major changes in the activated pathways were found after 6 hours and 12 hours of VEGF treatment, but increased activation of UPR and new activation of the Rap1 signaling and PI3K-Akt signaling pathways were noted. UPR signaling has been recently highlighted due to its involvement in HIF1 $\alpha$-mediated VEGF expression and angiogenesis in cancers. These findings indicate that angiogenic pathways are concomitantly and strongly induced promptly after VEGF treatment and remain activated for an extended period. Overall, the TNF, TGFBR2, NF-KB, and MAP kinase signaling pathways showed early activation after VEGF treatment, whereas the canonical Wnt, Notch, Rap1, and PI3K-Akt signaling pathways showed relatively late activation (Supplementary Table 1).

At early time points (20 and 45 minutes after HMGB-1 treatment), 28 genes were upregulated by HMGB-1, and these were related to inflammatory response, cell adhesion, transcription, and signal transduction, which are pathways activated in the early process of HMGB-1 binding to its receptor. After 2 hours of HMGB-1 treatment, 49 genes were upregulated, and some of which belonged to angiogenic pathways such as cell proliferation and hypoxia. Our functional annotation analysis further showed that 85 and 182 genes were upregulated 6 and 12 hours after HMGB-1 treatment, respectively, and these were closely related to both direct and indirect angiogenic pathways, such as response to wounding, blood vessel development, cell proliferation, apoptosis, vasculogenesis, angiogenesis, and inflammatory response. These findings suggest that prolonged exposure to HMGB-1 initiates strong activation of direct angiogenic pathways including migration, proliferation, and sprouting in ECs, accompanying early activation of the inflammatory response, an indirect angiogenic pathway. Although HMGB-1 and VEGF commonly induced EC activation and subsequent angiogenesis, HMGB-1-mediated activation of ECs was shown to be quite different from VEGF-mediated activation in terms of the order of pathway activation and the upregulated genes involved (Supplementary Table 1).

\section{HMGB-1 induces novel angiogenic and oncogenic genes}

Integrative gene ontology analysis was performed using the 245 genes upregulated more than 1.5-fold during at least one time point after HMGB-1 treatment (Fig. 2C). Of these, 99 genes were upregulated by both HMGB-1 and VEGF treatment. Ontology analysis of the 146 genes specifically upregulated by HMGB-1 revealed no significant angiogenesis-relevant pathways, while the 99 genes upregulated by both HMGB-1 and VEGF were closely related to direct and indirect angiogenic pathways, such as response to wounding, inflammatory response, angiogenesis, cell chemotaxis, and cell proliferation. To analyze the time-dependent expression changes of each gene, we further selected the 83 genes upregulated more than 1.5-fold during at least two time points (Fig. 2C). Importantly, expression of most genes $(n=62)$ started to increase after 6 hours of HMGB-1 treatment and remained upregulated until 12 hours of HMGB-1 treatment (Fig. 2D). Out of the 83 genes, 59 were specifically upregulated by HMGB-1, and some of these genes were related to angiogenic pathways including inflammatory response, cell proliferation, epidermal growth factor receptor (EGFR) signaling, and cell-cell junction. Specifically, ABL2, AFAP1L2, APOL3, CEACAM1, CHST1, CLDN5, KIT, PLA2G4C, RHBDF2, TNFRSF21, GJA4, and VIPR1 are reported to play a role in physiological or pathological angiogenesis (Supplementary Table 2). We further 
selected $A B L 2, C E A C A M 1, V I P R 1$, and KIT to demonstrate the angiogenic roles of the HMGB-1 specifically upregulated genes. Wound healing and tube formation assays were performed after HMGB-1 treatment when HMGB-1 specifically upregulated genes were inhibited by RNAi. Efficient downregulation of each gene was confirmed by qRT-PCR in ECs transfected with respective RNAi (Supplementary Fig. S3A). The wound healing assay showed that the wound healing process promoted by HMGB-1 treatment was significantly dysregulated by inhibition of $A B L 2$, CEACAM1, and VIPR1 and most potently by inhibition of KIT (Supplementary Fig. S3B and S3C). The tube formation assay also showed a similar result in which HMGB-1 treated ECs barely formed capillary-like structures when HMGB-1 specifically up-regulated genes were inhibited. Consistent with the wound healing assay results, inhibition of KIT most dramatically led to a reduction in capillary formation of ECs (Supplementary Fig. S3D and S3E).

Out of the 83 genes, the remaining 24 genes were upregulated in both HMGB-1-treated and VEGF-treated ECs, and some of these were related to response to wounding, angiogenesis, cell adhesion, and cell chemotaxis, which were similarly identified in the analysis of the 99 genes upregulated by both HMGB-1 and VEGF during at least one time point. Specifically, CCL2, EPHB1, PCDH12, EGR1, and CD34 are involved in physiological or pathological angiogenesis (Supplementary Table 2). In contrast, 551 and 283 genes were found to be specifically upregulated (more than 1.5-fold) by VEGF during at least one time point and at least two time points, respectively. As expected, most of these genes were related to well- documented VEGF-induced direct and indirect angiogenic pathways, including cell migration, response to wounding, UPR signaling, PDGFR signaling, cell adhesion, cell proliferation, apoptosis, MAP kinase signaling, PI3K-Akt signaling, VEGFR signaling, Wnt signaling, TGFBR2 signaling, TNF signaling, Rap1 signaling, and NF-KB signaling (Fig. 2C).

\section{DISCUSSION}

Angiogenesis is the physiological or pathological proliferation of capillaries from pre-existing blood vessels. Importantly, pathological conditions such as tumor growth and metastasis accompany the dysregulation of angiogenesis, which can be induced by several major molecular mechanisms including hypoxia and abnormally activated VEGFR signaling (19). Although a significant fraction of patients with cancer respond to VEGF-targeted therapies, clinical use of VEGF blockers results in different outcomes according to cancer type $(20,21)$. Moreover, angiogenesis in patients with advanced cancer is regulated not only by VEGF but also by other proangiogenic molecules, making it difficult to block angiogenesis using VEGF inhibitors (18). HMGB-1, a cytokine broadly overexpressed in most cancers, is known to be a potent inducer of angiogenesis and has been studied as a target molecule for anti-angiogenic therapy $(7,11)$. Under both physiological and pathological conditions, HMGB-1 binds to RAGE, and the HMGB-1/RAGE axis activates the MAP kinase/NF- $\mathrm{kB}$ and CDC42/Rac pathways, contributing to EC proliferation, migration, and sprouting (7). However, most of these findings were derived from unrelated in vitro experiments, and the molecular mechanism by which HMGB-1 activates ECs and subsequently induces angiogenesis remains unclear.

In this study, we performed time-dependent gene expression analysis of ECs to characterize HMGB-1- or VEGF-mediated changes in the transcriptome. Ontology analysis showed that genes upregulated by VEGF mostly belonged to conventional VEGF/VEGFR axis-mediated pathways such as cell proliferation, migration, death, angiogenesis, and hypoxia. Most of these angiogenic pathways were activated promptly after VEGF treatment and showed increased activation over time. HMGB-1-induced angiogenic pathways mostly overlapped with those activated by VEGF, even though the upregulated genes in these common pathways differed. Moreover, angiogenic pathways began to be highly activated after 6 hours of HMGB-1 treatment. These findings suggest that HMGB-1-induced angiogenesis might require a longer period of exposure than VEGF-induced angiogenesis and involve some HMGB-1-specific angiogenic factors.

We found that 83 genes were upregulated by HMGB-1 during at least two time points, and most of these were continuously upregulated from 6 to 12 hours after HMGB-1 treatment. Out of these 83 genes, 24 were upregulated by both HMGB-1 and VEGF, and most of these were closely related to angiogenic pathways including response to wounding, angiogenesis, cell adhesion, and cell chemotaxis, suggesting that HMGB-1 at least partially induces angiogenesis in a VEGF-like manner. Based on the known functions of these 24 genes, 13 genes were related to physiological or tumor angiogenesis. Notably, four genes (CCL2, EGR1, EPHB1, and PCDH12) are reported to independently promote angiogenesis in vitro or in vivo. CCL2 accelerates cancer metastasis by promoting angiogenesis, and its expression is also regulated by the HMGB-1-mediated activation of TLR4 signaling $(22,23)$. EGR1 is a well-known angiogenic factor whose expression is reportedly regulated by HMGB-1/RAGE signaling and VEGF/ VEGFR signaling $(24,25)$. In addition, EGR1 is reportedly involved in endothelial cell growth, tumor angiogenesis, and tumor growth and has even been considered as an antiangiogenic therapeutic target (25). EPHB1, another well-known angiogenic factor, is expressed in the developing vasculature and promotes angiogenesis (26). $\mathrm{PCDH} 12$ is required for normal angiogenesis and is highly expressed in angiogenic ECs (27). We here report the relationships between HMGB-1 and the genes $E P H B 1$ and $P C D H 12$ for the first time.

The characterization of the 59 genes that were specifically upregulated by HMGB-1 during at least two time points is crucial, as these genes explain HMGB-1-specific angiogenesis. We found that 12 genes were functionally related to 
angiogenesis, and four (ABL2, CEACAM1, KIT, and VIPR1) are reported to directly induce in vitro and in vivo angiogenesis. $\mathrm{ABL} 2$, an oncogenic non-receptor tyrosine kinase, is reported to be critical for the survival and activation of ECs (28). CEACAM1, a cell adhesion protein, functions as an activator in both physiological and tumor angiogenesis by inducing blood vessel remodeling through EC differentiation (29). KIT is a well-known oncogenic receptor tyrosine kinase with reported involvement in physiological and tumor angiogenesis (30). VIPR1, a vasoactive intestinal peptide receptor, plays a major role in progression and angiogenesis in a number of cancers $(31,32)$. In our wound healing and tube formation assays, we demonstrated that inhibition of these four genes dramatically disturbed HMGB-1 mediated angiogenic features in ECs, which suggests that HMGB-1 specifically upregulated genes play critical roles in evoking angiogenic features in ECs. In this context, HMGB-1-mediated upregulation of these four genes could cause VEGF independent angiogenesis, which might explain drug resistance to anti-angiogenesis therapy based on VEGF inhibitors in cancer patients.

HMGB-1 induces the upregulation of HMGB-1-specific angiogenic factors, accompanying VEGF-like upregulation of some conventional angiogenic factors. This suggests that HMGB-1 can independently induce angiogenesis and also synergistically enhance VEGF-mediated angiogenesis. In future studies, the angiogenic genes induced by HMGB-1 should be further characterized to understand how they contribute to angiogenesis in physiological and tumor microenvironments and how to inhibit abnormal angiogenesis in various cancers.

\section{MATERIALS AND METHODS}

\section{Cell culture and treatment}

Primary human umbilical vein endothelial cells (HUVECs) were isolated from human placental microvessels according to previous methods $(33,34)$ and maintained in M199 (GE Healthcare Life Sciences, Logan, UT, USA) supplemented with $20 \%$ fetal bovine serum (FBS), 1\% penicillin/streptomycin, 10 $\mu \mathrm{g} / \mathrm{ml}$ bFGF (Sigma, St. Louis, MO, USA), and 2500 units/ml heparin (Sigma). This study was approved by the Institutional Review Board of Yonsei University College of Medicine (No.12-genebank-11). Written informed consent was obtained from all patients. HUVECs between the second and fifth passages were used. HUVECs were treated with recombinant HMGB-1 (ProSpec-Tany TechnoGene Ltd., Ness Ziona, Israel) at $1 \mu \mathrm{g} / \mathrm{ml}$ and recombinant VEGF (ProSpec) at $50 \mathrm{ng} / \mathrm{ml}$ for 20 minutes, 45 minutes, 2 hours, 6 hours, or 12 hours.

\section{Gene expression microarray}

For DNA microarray hybridization, RNA was extracted from HUVECs using TRIzol (Invitrogen Life Technologies, Carlsbad, CA, USA). RNA was pooled by mixing equal amounts of total RNA, and biotin-labeled cRNA targets were synthesized from $1.5 \mu \mathrm{g}$ of total RNA. Double-stranded cDNA synthesis was performed using the Illumina ${ }^{\circledR}$ TotalPrep RNA Amplification Kit (Illumina, Inc., San Diego, CA, USA), while biotinUTP-labeled antisense RNA was transcribed in vitro using the Ambion MEGAscript kit (Ambion Life Technologies, Carlsbad, CA, USA). All steps of the labeling procedure were performed according to the manufacturers' protocols. Microarray experiments were conducted on the HumanHT-12 v4 Sentrix Expression BeadChip (Illumina) containing 47,231 probes representing 31,332 annotated genes.

\section{Gene enrichment, functional annotation, and statistical analyses}

Data are expressed as mean \pm standard deviation (SD). P $<$ .05 was considered significant. Student's t-tests were performed using SPSS for Windows (SPSS Inc., Chicago, IL, USA). DAVID software (Database for Annotation, Visualization and Integrated Discovery, v6.8; http://david.abcc.ncifcrf.gov) was used for pathway and gene ontology analysis.

\section{ACKNOWLEDGEMENTS}

This work was supported by the Yonsei University College of Medicine (grant number 6-2014-0132).

\section{CONFLICTS OF INTEREST}

The authors have no conflicting interests.

\section{REFERENCES}

1. Lotze MT and Tracey KJ (2005) High-mobility group box 1 protein (HMGB1): nuclear weapon in the immune arsenal. Nat Rev Immunol 5, 331-342

2. Yusein-Myashkova S, Ugrinova I and Pasheva E (2016) Non-histone protein HMGB1 inhibits the repair of damaged DNA by cisplatin in NIH-3T3 murine fibroblasts. BMB Rep 49, 99-104

3. Tas SW, Maracle CX, Balogh E and Szekanecz Z (2016) Targeting of proangiogenic signalling pathways in chronic inflammation. Nat Rev Rheumatol 12, 111-122

4. Hori O, Brett J, Slattery T et al (1995) The receptor for advanced glycation end products (RAGE) is a cellular binding site for amphoterin. Mediation of neurite outgrowth and co-expression of rage and amphoterin in the developing nervous system. J Biol Chem 270, 25752-25761

5. Park JS, Svetkauskaite D, He Q et al (2004) Involvement of toll-like receptors 2 and 4 in cellular activation by high mobility group box 1 protein. J Biol Chem 279, 73707377

6. Ivanov S, Dragoi AM, Wang X et al (2007) A novel role for HMGB1 in TLR9-mediated inflammatory responses to CpG-DNA. Blood 110, 1970-1981

7. Yang S, Xu L, Yang T and Wang F (2014) High-mobility group box-1 and its role in angiogenesis. J Leukoc Biol 95, 563-574 
8. Biscetti F, Ghirlanda G and Flex A (2011) Therapeutic potential of high mobility group box-1 in ischemic injury and tissue regeneration. Curr Vasc Pharmacol 9, 677-681

9. Sims GP, Rowe DC, Rietdijk ST, Herbst R and Coyle AJ (2010) HMGB1 and RAGE in inflammation and cancer. Annu Rev Immunol 28, 367-388

10. Jung B, Kang H, Lee W et al (2016) Anti-septic effects of dabrafenib on HMGB1-mediated inflammatory responses. BMB Rep 49, 214-219

11. Tang D, Kang R, Zeh HJ and Lotze MT (2010) High-mobility group box 1 and cancer. Biochim Biophys Acta 1799, 131-140

12. Maeda S, Hikiba Y, Shibata W et al (2007) Essential roles of high-mobility group box 1 in the development of murine colitis and colitis-associated cancer. Biochem Biophys Res Commun 360, 394-400

13. Kim SW, Jin Y, Shin JH et al (2012) Glycyrrhizic acid affords robust neuroprotection in the postischemic brain via anti-inflammatory effect by inhibiting HMGB1 phosphorylation and secretion. Neurobiol Dis 46, 147-156

14. Kang R, Zhang Q, Zeh HJ, Lotze MT and Tang D (2013) HMGB1 in cancer: good, bad, or both? Clin Cancer Res $19,4046-4057$

15. Weidner N, Semple JP, Welch WR and Folkman J (1991) Tumor angiogenesis and metastasis-correlation in invasive breast carcinoma. N Engl J Med 324, 1-8

16. Sasahira T, Kirita T, Bhawal UK et al (2007) The expression of receptor for advanced glycation end products is associated with angiogenesis in human oral squamous cell carcinoma. Virchows Arch 450, 287-295

17. van Beijnum JR, Dings RP, van der Linden E et al (2006) Gene expression of tumor angiogenesis dissected: specific targeting of colon cancer angiogenic vasculature. Blood $108,2339-2348$

18. Carmeliet P and Jain RK (2011) Molecular mechanisms and clinical applications of angiogenesis. Nature 473, 298-307

19. Carmeliet $P$ and Jain RK (2000) Angiogenesis in cancer and other diseases. Nature 407, 249-257

20. Jain RK, Duda DG, Willett CG et al (2009) Biomarkers of response and resistance to antiangiogenic therapy. Nat Rev Clin Oncol 6, 327-338

21. Bergers $G$ and Hanahan D (2008) Modes of resistance to anti-angiogenic therapy. Nat Rev Cancer 8, 592-603

22. Bonapace L, Coissieux MM, Wyckoff J et al (2014) Cessation of CCL2 inhibition accelerates breast cancer metastasis by promoting angiogenesis. Nature 515,
130-133

23. Cai J, Yuan H, Wang $\mathrm{Q}$ et al (2015) HMGB1-Driven Inflammation and Intimal Hyperplasia After Arterial Injury Involves Cell-Specific Actions Mediated by TLR4. Arterioscler Thromb Vasc Biol 35, 2579-2593

24. Lv B, Wang H, Tang Y, Fan Z, Xiao X and Chen F (2009) High-mobility group box 1 protein induces tissue factor expression in vascular endothelial cells via activation of NF-kappaB and Egr-1. Thromb Haemost 102, 352-359

25. Liu L, Tsai JC and Aird WC (2000) Egr-1 gene is induced by the systemic administration of the vascular endothelial growth factor and the epidermal growth factor. Blood 96, 1772-1781

26. Kojima T, Chang JH and Azar DT (2007) Proangiogenic role of ephrinB1/EphB1 in basic fibroblast growth factor-induced corneal angiogenesis. Am J Pathol 170, 764-773

27. Rampon C, Bouillot S, Climescu-Haulica A et al (2008) Protocadherin 12 deficiency alters morphogenesis and transcriptional profile of the placenta. Physiol Genomics 34, 193-204

28. Song G, Li Y and Jiang G (2012) Role of VEGF/VEGFR in the pathogenesis of leukemias and as treatment targets (Review). Oncol Rep 28, 1935-1944

29. Gerstel D, Wegwitz F, Jannasch K et al (2011) CEACAM1 creates a pro-angiogenic tumor microenvironment that supports tumor vessel maturation. Oncogene 30, 42754288

30. Patruno R, Marech I, Zizzo N et al (2014) c-Kit expression, angiogenesis, and grading in canine mast cell tumour: a unique model to study c-Kit driven human malignancies. Biomed Res Int 2014, 730246

31. Valdehita A, Carmena MJ, Collado B, Prieto JC and Bajo AM (2007) Vasoactive intestinal peptide (VIP) increases vascular endothelial growth factor (VEGF) expression and secretion in human breast cancer cells. Regul Pept 144, 101-108

32. Tang B, Yong X, Xie R, Li QW and Yang SM (2014) Vasoactive intestinal peptide receptor-based imaging and treatment of tumors (Review). Int J Oncol 44, 1023-1031

33. Ugele B and Lange F (2001) Isolation of endothelial cells from human placental microvessels: effect of different proteolytic enzymes on releasing endothelial cells from villous tissue. In Vitro Cell Dev Biol Anim 37, 408-413

34. Kacemi A, Challier JC, Galtier M and Olive G (1996) Culture of endothelial cells from human placental microvessels. Cell Tissue Res 283, 183-190 